\title{
To Reduce the Error Probability of Aggressive Packet Combining Scheme in the Markov's Three State Model
}

\author{
Preeti Sharma \\ M-Tech CSE \\ National institute of technology \\ Arunachal Pradesh, Yupia
}

\author{
Balkrishna Choubey \\ Asst.proff. ECE \\ SSIPMT/CSVTU \\ Raipur, Chhattisgarh
}

\begin{abstract}
For the reliable communication it is necessary to successfully transmit data. Typical aggressive packet combining technique is a very efficient technique in the data communication for the error detection and correction in wireless communication. But there are some demerits of typical APC. In this new technique some challenges of APC are overcome by using Markov model of the channel. Markov's law [1] suggests the possibility of channel state is three; Good state, moderate state and bad state. For three states, different methods areimplemented. When the channel is in good state original data is sent. When the channel is moderate state then bit combination method is used and for bad state half byte packet reversal method is used.
\end{abstract}

\section{General Terms}

Proposed technique, literature review, packet reverse packet combining scheme

\section{Keywords}

Typical aggressive packet combining scheme, markov's three state models, half byte packet reversal, bit-combination technique.

\section{INTRODUCTION}

A major challenge for the researcher is to successfully transmit the data from source to destination. Two major techniques are used for error detection and correction in data communication. These two techniques are FEC (forward error correction) and BEC (backward error correction). Aggressive packet combining (APC) scheme is one of the example of FEC.APC proposed by Leung [2]. APC is very efficient technique for data transmission from sender to receiver. Several modifications have been done in the APC [3-9] to improve its performance. InAPC, three copies of the original packet are transmitted to the receiver. In the Markov's three state model $[10,11]$ there are three states. One is good state, second is moderate state and third state is bad state. If state is good then copies are sent as conventional APC. When the state is moderate state then bit combination method is apply. If state is bad state, then copies are sent by some modified technique (half byte packet reversal).This modification is shown in the proposed technique.

\section{LITRATURE REVIEW}

\subsection{Packet Reversed Packet Combining Technique}

The idea behind packet reverse packet combining (PRPC) $[12,13]$ is that the receiver when receives an erroneous packet and requests for retransmission of another copy without discarding the first erroneous copy, the transmitter transmits a bit reversed packet of original packet. The idea and operation of PRPC is illustrated with several examples below.
Let "00110101" be the original packet. On first transmission receiver receive erroneous packet i.e. "00111101" (error at $5^{\text {th }}$ bit location from the left) thus, Retransmission of packet is requested from receiver to transmitter. Transmitter retransmits second copy after applying the Packet reverse packet combining scheme (i.e. LSB of original packet is now MSB of bit reversed packet and vice versa) on original packet i.e. "00110101". Receiver again receives erroneous copy "00111101" (bit error in $5^{\text {th }}$ bit location from the left). Receiver applies the same phenomenon of PRPC at received copy to decode (decoded result is "00100101"). Now, packet combining technique is performed at the received two copies.

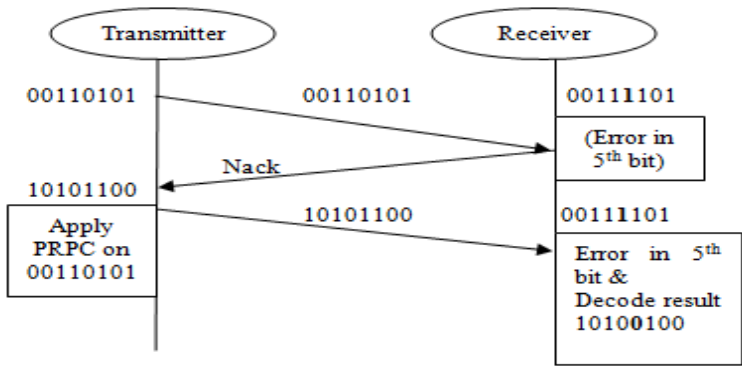

Fig 1- packet reversed packet combining technique

In the example, XOR of first and reversed second copy will result,

\begin{tabular}{l} 
First received copy $=00111101$ \\
Second received copy $=00100101$ \\
\hline XOR operation $=00011000$
\end{tabular}

Error occurs at 4 th $\& 5$ th bit position from left. Now apply the Brute Force method to correct the error. The PRPC scheme will be able to correct single bit errors by using two consecutive erroneous packets when error occurs at the same location.

\subsection{Aggressive packet combining scheme}

Aggressive packet combining scheme is a modification of MjPc (Majority Packet Combining) [14]. In APC three copies of the original packet is sent from source to destination. If received copies are erroneous then, bit wise majority logic is applied on the three copies at receiver side. Let "10011" be the original packet which is to be transmitted from source to destination.

Example: Let the original packet is 10011 .

Majority logic:

Transmitter $\longrightarrow$ Receiver 


$\begin{array}{ll}10011 & 10111 \\ 10011 & 01011 \\ 10011 & 10010\end{array}$

Majority logic =

10011(original packet)

But APC fails when error occurs in the same bit location of the received three copies as shown in table.

Table 1: APC fails to correct error when occur in same location of all the received copies for the packet 1011.

\begin{tabular}{|l|l|l|l|l|l|}
\hline $\begin{array}{l}\text { Receive } \\
\text { d copies }\end{array}$ & $\begin{array}{l}\text { Bitwise } \\
\text { Majorit } \\
\text { Voting }\end{array}$ & $\begin{array}{l}\text { Error } \\
\text { detecte } \\
\mathrm{d}\end{array}$ & $\begin{array}{l}\text { Least } \\
\text { reliabl } \\
\text { e bits }\end{array}$ & $\begin{array}{l}\text { Searchin } \\
\text { g correct } \\
\text { bit } \\
\text { pattern }\end{array}$ & $\begin{array}{l}\text { Error } \\
\text { detecte } \\
\mathrm{d}\end{array}$ \\
\hline 1010 & 1010 & yes & $\begin{array}{l}2^{\text {nd }} \\
\text { from } \\
\text { left }\end{array}$ & 1110 & yes \\
1110 & & & & & \\
0010 & & & & & \\
\hline
\end{tabular}

\section{PROPOSED TECHNIQUE}

In the typical Aggressive packet combining scheme (APC) if the two or more error occurs in particular bit position of the three copies of the same packet, then typical APC fails. Markov's model describes that there are three states of the network. First state is the good state, second state is moderate state and third state is bad state. We will assume the channel to be good when no packet is received erroneous, channel to be moderate when atleast one packet is received erroneous and channel to be bad when more than twopackets is received erroneously. In the good state original packet is sent to the receiver. When the receiver receives the error in atleast one or two packets then this state is moderate state. In the moderate state channel, ifreceiver receives NACK for atleast one packet then the packetis retransmittedafter implementing bitcombination method. When receiver receives multiple erroneous packets then it is called the bad state condition. In the bad state condition transmitter implements half byte packet reversal technique and then send it to the receiver. A receiver decodes the packets and appliesAPC to get the correct copy. Initially it is assumed that the channel is in good condition and the following procedures are performed:

Step 1: Transmitter transmits the three packets at a time; P1, $\mathrm{P} 2$ and $\mathrm{P} 3$.

Step 2: Receiver receives all packets without any error (i.e. Good state). Thus ACK message is transmitted back to transmitter.

Step 3:Again Transmitter transmits three packets P4, P5 and P6. Now, Error is detected at packet 5 and packet 6 . Thus, it is understood that channel is switched to moderate state.

Step 4: NACK message is transmitted towards transmitter for erroneous packet (P5 \& P6) and request for retransmission.

Step 5: At moderate channel state, Bit combination method is applied at previously received erroneous packet (i.e. P5 \& P6) and retransmit towards receiver.

Step 6: Receiver receivers' packets both retransmitted one (i.e. packet after applying bit combination P5 \& P6) and the original one (P7). APC technique is applied at the received packets.
Step 7: Bad state, when the entire received packet(i.e. P8, P9 \& P10) areerroneously received.

Step 8: NAck message is send to the transmitter and request for retransmission.

Step 9: Before retransmission Half byte packet reversal is applied. APC technique is done at receiver side.

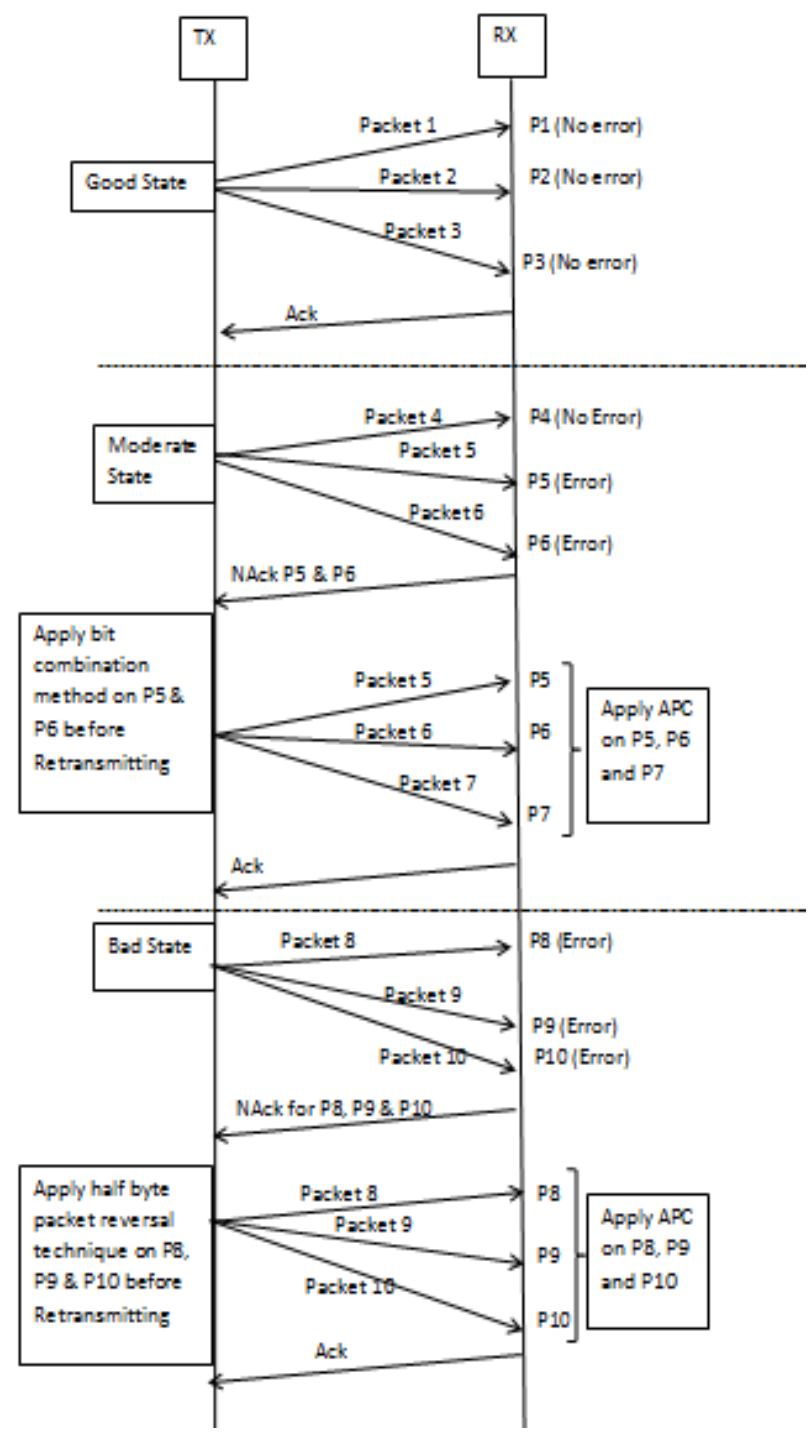

Fig.2: Proposed technique diagram

Example 1: Good State (original packet 11001)

If all the received packets (P1 P2 \& P3) are correct then apply the majority voting as typical APC. This is the good state condition

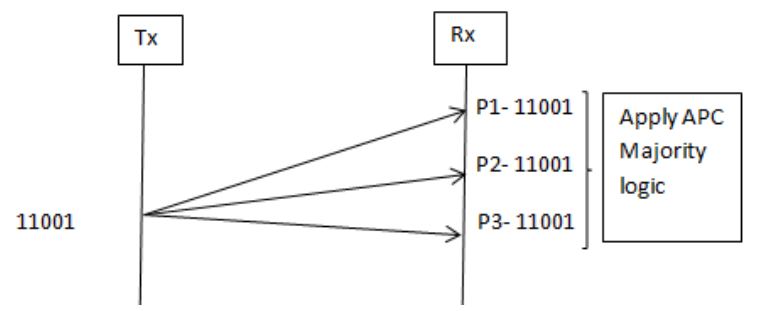

Fig 3: Good state condition 
Majority voting:

\begin{tabular}{ll} 
Transmitter & Receiver \\
P1C1-11001 & 11001 \\
P1C2-11001 & 11001 \\
P1C3-11001 & 11001 \\
\hline Majority logic $=$ & 11001 (correct packet)
\end{tabular}

Example 2: Moderate State

Here original packet " 11001100 " is transmitted. AT receiver side two packets are received erroneously i.e. "P511011100 "(error at $4^{\text {th }}$ position) \&"P6-11001000" (error at $6^{\text {th }}$ position). If one or two packets are received erroneously, then it is known as the moderate state. Negative acknowledgement message are send for those packet which was received erroneously. While responding to negative acknowledgement message, transmitter retransmitsafter applying the bit combination method on those packets which was received erroneously with the next packet which is to be transmitted as show in Figure 5, 6 and 7.

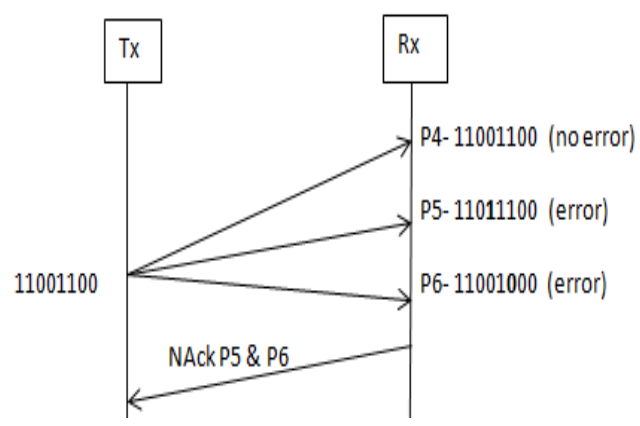

Fig 4: Moderate state condition

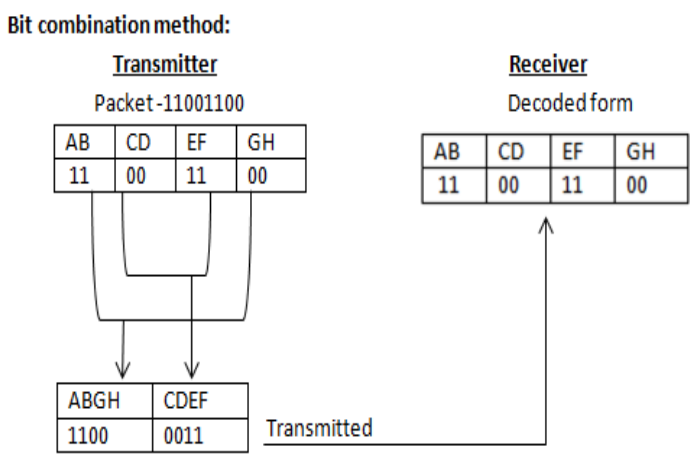

Fig 5: Bit combination method

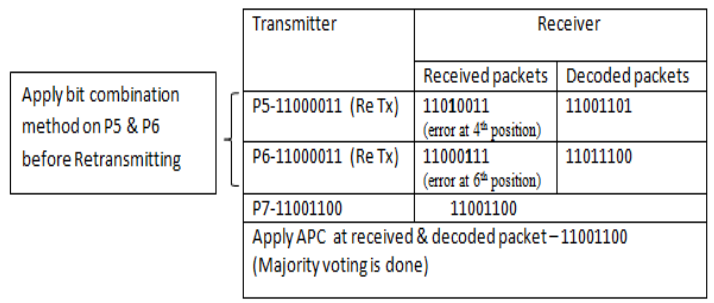

Fig 6- Retransmitting of Moderate state condition

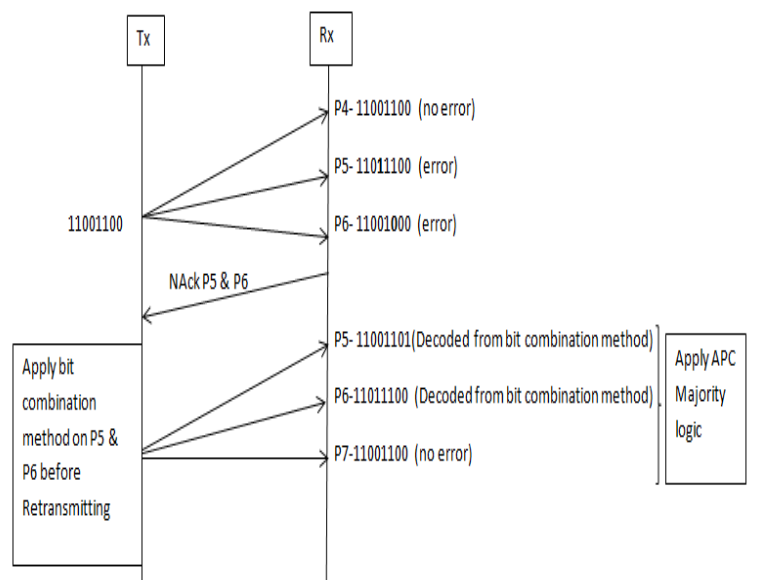

Fig.7: Moderate state condition

Example 3: Bad State

If all three packets are erroneously received then it is bad state condition.

Transmitter transmit back negative acknowledgment message and request for retransmits the packets. This

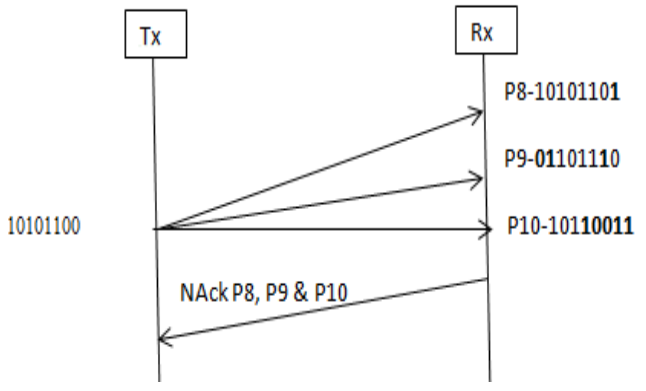

Fig 8: Bad state condition

In the bad state condition, transmitter retransmitted the packets afterapplying half byte packet reversal[12] technique.In half byte technique the packets are dividing into half part (i.e. MSB and LSB) then reversethe half byte packet.

$\begin{array}{lll}\text { Half byte reverse technique } & \\ \text { Original-10101100 } & \text { Divide into two parts } & \text { reverse the half byte packets } \\ 10101100 \longrightarrow & 0101 & 0011\end{array}$

Fig. 9:Half byte reversal technique.

Retransmitting of packet is done by half byte packet reversal technique.

The original copy is 10101100 . After apply the half byte packet reversal. These half packets are sent 3 times individually. (MSB - 010101010101$)$ \& (LSB -0011 0011 0011)

Step4: receiver received these copies and applies the majority voting. And then decode them. Received copies are - (MSB$010101101101)$ \& (LSB- 101101110011$)$

Apply the bit by bit majority voting 


\begin{tabular}{|l|l|}
\hline MSB - & LSB- \\
0101 & 1011 \\
0110 & 0111 \\
1101 & 0011 \\
\hline 0101 & 0011 \\
\hline
\end{tabular}

Now receiver decodes this copy and gets the correct copy.

\begin{tabular}{|c|c|}
\hline MSB & LSB \\
0101 & 0011 \\
$\downarrow$ & $\downarrow$ \\
1010 & 1100 \\
\hline \multicolumn{2}{|}{} \\
10101100( corrected copy) \\
\hline
\end{tabular}

\section{MATHEMATICAL ANALYSIS}

In our proposed technique let us consider the channel have and three states. First is the good state condition second is the moderate state condition and third is bad state condition The probability that the channel is in good, moderate and bad state are respectively $\mathrm{p} 0, \mathrm{p} 1$, and $\mathrm{p} 2$. It is a reasonable assumption that in a session the channel will be $33 \%$ in $P 0$, $33 \%$ in $\mathrm{p} 1$, and $33 \%$ inp2 state in normal environment.

Probability of packet error is given by:

$P e=1-(1-\alpha) N$,

Where $\alpha$ is a bit error rate. $\mathrm{N}=$ no. of bits in packet

In our proposed scheme when the half byte packet reversal is used,[13] probability of packet error is:

\section{$P e / 2=1-(1-\alpha) \mathrm{N} / 2$}

\section{CONCLUSION}

In this new technique we can conclude that, if we are using the multipath technique in markov's three state models the error probability will be reduced. And if the channel is in bad state half byte packet reversal are used this technique also reduced the packet error rate and increase the throughput.

\section{ACKNOWLEDGEMENT}

I would like to expand my research paper in practically.Special thanks to whoever are helping me for this research paper, my SUPERVISOR, H.O.D and faculty member of National Institute of Technology, Arunachal Pradesh .Without their active guidance, motivation and help, we would not have made headway in this research paper.

\section{REFERENCES}

[1] Attilio Di Marco, Ann Arbor, "A Semi-Markov Model Of A Three-State Generating Unit", IEEE Power Engineering Society, New York, N.Y., January 30February 4, 1972.
[2] Yiu-Wing Leung, "Aggressive Packet Combining for error control in wireless networks", trans. Comm vol. E83, No 2 Feb' 2000, pp38-385

[3] Yaka Bulo, Swarnendu K Chakraborty, C T Bhunia "New protocol of Aggressive Packet Combining scheme",lnternational Journal ofComputer Applications(0975-8887), vol. 85, No. 6, January 2014.

[4] Preeti sharma,Yaka bulo, "logical aggressive packet combining scheme with post reception selection combining scheme for wireless network" ieee confrence,ICCSP-2015.

[5] Yaka Bulo,C. T. Bhunia, "New Protocol of AggressivePacket Combining Scheme: An extension to throughput comparisonll, International Journal of Computer Applications,USA (0975 8887) Volume 113 No. 5, pp- 5-8 March 2015.

[6] S. K. Chakraborty, R. S. Goswami, A. Bhunia, C. T. Bhunia, "Investigation of Two New Protocols of Aggressive Packet combining scheme in achieving better throughput", Journal of the Institute of Engineers (India) B, Springer,DOI: 10.1007/ s40031 -014-0135-7.

[7] Yang Saring, "Four New Protocols for achieving BetterCorrection Capability of APC SchemeIEEE ICCSP 2015 conference.

[8] C. T. Bhunia, "Several Modifications of Aggressive Packet Combining Scheme for Wireless Network", United Nations Educational, Scientific and Cultural Organization and International Atomic Energy Agency, THE ABDUS SALAM INTERNATIONAL CENTRE FOR THEORETICAL PHYSICS, 2010.

[9] Yang Saring, Yaka Bulo, C T Bhunia, "New Modifications of Aggressive Packet Combining Scheme with improved performance," International Journal of Applied Engineering Research, ISSN 0973 - 4562, Volume 10, Number 17 (2015) pp. 37610- 37615.

[10] Ming Li, Mingchuan Yang"Three-State Semi-Markov Channel Model for HAPHighSpeed Train Communication Link “ 2011 6th International ICST Conference on Communications and Networking in China (CHINACOM)

[11] Lu Lu Institute of Communication Engineering, PLA University of Science and Technology Nanjing, 210007, Chinakatherine10@163.comAnalysis of Channel Model for GEO Satellite MobileCommunication System National Conference on Information Technology and Computer Science (CITCS 2012).

[12] C T Bhunia,"Packet Reversed Packet Combining Scheme", Proc. IEEE Computer soc., CIT' 07, Aizu University, Japan, pp 447-451

[13] Yaka Bulo,Preeti Sharma“'Improving Error Correction Capability of Aggressive Packet Combining Scheme by using Half-Byte Packet Reverse Technique and EvenOdd Selection Method"International Journal of Computer Applications (0975 -8887)Volume137No.2,March-2016. 